\title{
Spatial solitons in nonlinear photonic crystals
}

\section{Corney, Joel Frederick; Bang, Ole}

\section{Published in:}

Nonlinear Optics: Materials, Fundamentals, and Applications

Link to article, DOI:

10.1109/NLO.2000.883597

Publication date:

2000

\section{Document Version}

Publisher's PDF, also known as Version of record

Link back to DTU Orbit

Citation (APA):

Corney, J. F., \& Bang, O. (2000). Spatial solitons in nonlinear photonic crystals. In Nonlinear Optics: Materials, Fundamentals, and Applications (pp. 122-124). https://doi.org/10.1109/NLO.2000.883597

\section{General rights}

Copyright and moral rights for the publications made accessible in the public portal are retained by the authors and/or other copyright owners and it is a condition of accessing publications that users recognise and abide by the legal requirements associated with these rights.

- Users may download and print one copy of any publication from the public portal for the purpose of private study or research.

- You may not further distribute the material or use it for any profit-making activity or commercial gain

- You may freely distribute the URL identifying the publication in the public portal

If you believe that this document breaches copyright please contact us providing details, and we will remove access to the work immediately and investigate your claim 


\title{
Spatial solitons in nonlinear photonic crystals
}

\author{
J. F. Corney and O. Bang \\ Department of Mathematical Modelling, Technical University of Denmark, \\ DK-2800 Lyngby, Denmark \\ Ph: +45 45253108, Fax: +45 45931235,jfc@imm.dtu.dk
}

\begin{abstract}
We study solitons in one-dimensional quadratic nonlinear photonic crystals with periodic linear and nonlinear susceptibilities. We show that such crystals support stable bright and dark solitons, even when the effective quadratic nonlinearity is zero.

(c) 2000 Optical Society of America

OCIS codes: (190.5530) Pulse propagation and solitons; (230.4320) Nonlinear optical devices; (190.4410) Nonlinear optics, parametric processes; (190.5940) Self-action effects
\end{abstract}

Modulating the linear properties of optical crystals constitutes the backbone of the rapidly developing field of photonics, which has focussed on engineering the dispersion curve of crystals to create photonic band-gaps and other features that would enable all-optical signal processing. However, the nonlinearity of a crystal can also be modulated, a fact which greatly extends the crystal's potential utility in photonic applications[1].

Parametric cascading processes in $\chi^{(2)}$ materials are of particular interest for the development all-optical components due to the fast, large nonlinearity. The efficiency of these processes depends on the use of phase matching techniques. Phase matching can be most efficiently achieved by periodically modulating the nonlinearity, a method known as quasi-phase matching (QPM)[2]. However, in creating a nonlinear grating in a material, a simultaneous linear grating[3] is often (intentionally or unintentionally) created, for example when implementing QPM gratings in GaAs/AlAs through quantum-well disordering[4].

For these reasons, we focus on manipulating both the linear and nonlinear properties of $\chi^{(2)}$ materials. In particular, we study the propagation of solitons in one-dimensional nonlinear photonic crystals in $\chi^{(2)}$ materials (slab waveguides), taking into consideration the modulation of both the linear and nonlinear susceptibilities. Building on previous studies, which find that simultaneous gratings may reduce the effective quadratic nonlinearity $[2,3]$ and that the nonlinear grating will induce cubic nonlinearities[5], we numerically find previously unknown soliton solutions to first-order equations for the averaged fields. In the case of vanishing effective quadratic nonlinearity (which could occur even in material with a huge intrinsic $\chi^{(2)}$ nonlinearity) analytic soliton solutions to the effective equations still exist, due to the induced cubic nonlinearities. We demonstrate that, against intuition, the crystal supports the stable propagation of both bright and dark parametric solitons in a medium with apparently no nonlinearity. This is analogous to the existence of solitons in stongly dispersion-managed fibres with no average dispersion.

We begin with normalised time-independent envelope equations for the fundamental $w$ and second harmonic $v$ components of the electric field $[6,7]$ :

$$
0=\left(i \frac{\partial}{\partial z}+\frac{1}{2} \frac{\partial^{2}}{\partial x}+\Xi^{(1)}(z)\right) w+\chi(z) w^{*} v e^{i \Delta k z} ; \quad 0=\left(i \frac{\partial}{\partial z}+\frac{1}{4} \frac{\partial^{2}}{\partial x}+2 \Xi^{(2)}(z)\right) v+\chi(z) w^{2} e^{-i \Delta k z}
$$

where $\Xi^{(j)}(z)$ is the normalised linear susceptibility at the fundamental $(j=1)$ and second harmonic $(j=2)$ frequencies, and $\chi(z)$ is the nonlinear grating. For wave numbers $k_{1}$ and $k_{2}$ of the fundamental and second harmonic waves, we define the wave-number mismatch as $\Delta k=\left(k_{2}-2 k_{1}\right)$, which is positive for normal dispersion and negative for anomalous dispersion. We consider gratings for forward-wave first-order quasiphase matching, so that the spatial frequency must be $K \simeq \Delta k$. This means that the grating period will be much larger than the optical period, thereby minimizing any Bragg reflection effects.

The case without the linear grating has been dealt with elsewhere[5]. We use the same technique to derive approximate equations for the average fields $w_{0}$ and $v_{0}$ in the more general situation considered here. We assume that the residual phase mismatch is small $(\beta=\Delta k-K \ll K)$ and that the Fourier components in $K$ of the fields change little over a grating period. The resulting expressions take into account the lowest-order perturbations, proportional to the grating wavelength $\Lambda=2 \pi /\left|K^{*}\right|$. They are

$0=\left(i \frac{\partial}{\partial z}+\frac{1}{2} \frac{\partial^{2}}{\partial x}\right) w_{0}+\rho w_{0}^{*} v_{0}+\gamma\left(\left|v_{0}\right|^{2}-\left|w_{0}\right|^{2}\right) w_{0} ; \quad 0=\left(i \frac{\partial}{\partial z}+\frac{1}{4} \frac{\partial^{2}}{\partial x}+\beta\right) v_{0}+\rho^{*} w_{0}^{2}+2 \gamma\left|w_{0}\right|^{2} v_{0}$ 
where

$$
\rho=\frac{2 i}{\pi}\left(d+\frac{2 d_{0}}{K}\left(\kappa^{(1)}-\kappa^{(2)}\right)\right) ; \quad \gamma=\frac{1}{K}\left(d_{0}^{2}+d^{2}\left(1-\frac{8}{\pi^{2}}\right)\right) .
$$

Here we have assumed a square grating, in which $d_{0}$ is the DC component of the nonlinear grating and $2 d$ is the modulation depth. The modulation depths of the linear grating are $2 \kappa^{(1)}$ and $2 \kappa^{(2)}$, as illustrated in Figure 1.
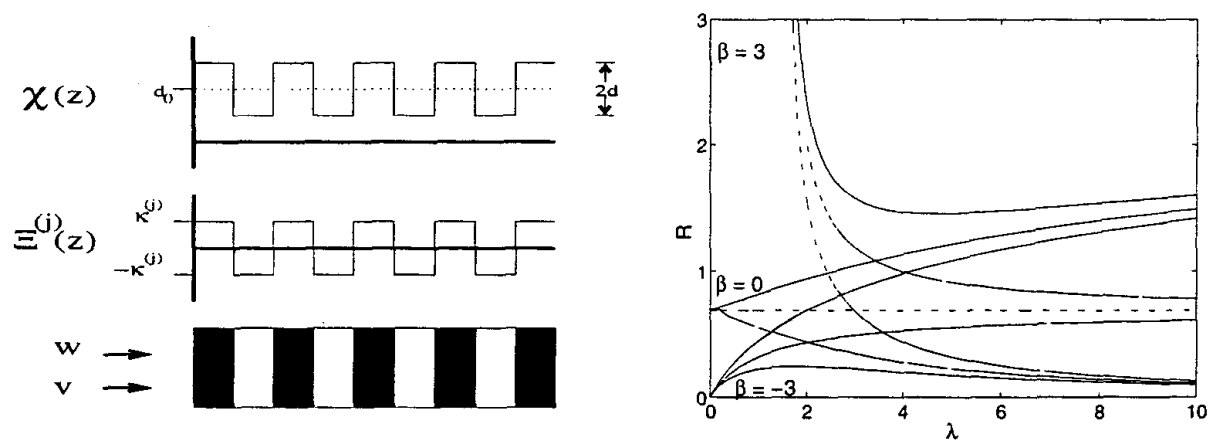

Fig. 1. LEFT: A square-wave modulation $\Xi^{(j)}(z)$ and $\chi(z)$ of the linear and nonlinear susceptibilities. RIGHT: Intensity ratio $R=\tilde{v}^{2}(0) / \tilde{w}^{2}(0)$ versus soliton parameter $\lambda$, for $\tilde{\gamma}>0$ (solid), $\tilde{\gamma}<0$ (dashed) and $\tilde{\gamma}=0$ (dotted).

Thus the nonlinear grating induces a cubic nonlinearity, consisting of a self-phase modulation in the fundamental field and cross-phase modulations in both fields. The cubic terms in each field may lead to either a focusing or a defocusing effect, depending on the sign of $\Delta k$ and the relative intensity of the fields. The size of the effective cubic nonlinearity depends only on the nonlinear grating, while the size of the effective quadratic nonlinearity depends on the interplay of linear and nonlinear grating effects.

The averaged equations [Eq. (2)] possess stable, localised solitary wave solutions, known as parametric solitons. In the absence of cubic terms in the equations, these solutions result from a cascading $\chi^{(2)}: \chi^{(2)}$ effect. The cubic terms can be relatively large in this system due to the average value $d_{0}$, and will affect the properties of the soliton solutions. We assume the form $w_{0}(x, z)=\frac{2}{\pi} \tilde{w}(x) e^{i \lambda z} /|\rho|, v_{0}(x, z)=\frac{2 i}{\pi} \tilde{v}(x) e^{2 i \lambda z} /|\rho|$, where $\lambda$ is the internal soliton parameter. In the equations for $\tilde{w}(x)$ and $\tilde{v}(x)$, the quadratic coefficient is a constant, while the cubic coefficient is $\tilde{\gamma}=4 \gamma /|\pi \rho|^{2}$. The slowly-varying approximation means that soliton solutions will be valid when the soliton period is longer than the grating period, i.e. $\lambda \ll|K|$.

Figure 1 plots the ratio of peak intensities versus $\lambda$, for three soliton families with $\beta=3, \beta=0$ and $\beta=-3$. In each case are shown the zeroth order solution $(\tilde{\gamma}=0)$ and the first-order solutions for both normal $(\tilde{\gamma}>0)$ and anomalous $(\tilde{\gamma}<0)$ dispersion, when $|\tilde{\gamma}|=0.0189$. The figure shows that the zeroth order approximation becomes increasingly inaccurate for large $\lambda$. Also, the solutions for a given $\tilde{\gamma}$ approach the same limiting value as $\lambda$ increases, regardless of the value of $\beta$. Calculations of the total power of the system reveal that there is a power threshold for the existence of solitons when $\beta>0$, which decreases for $\tilde{\gamma}>0$ and increases for $\tilde{\gamma}<0$. The $\tilde{\gamma}>0$ solutions have not been considered previously.

The soliton solutions of the approximate model [Eq. (2)] were tested by mapping them back to the variables $w$ and $v$, and launching them as the initial conditions into a numerical simulation of the field equations [Eq. (1)]. The subsequent evolution consists of rapid, small oscillations superimposed on the slow, large average beam. Properties of the propagating solutions were calculated by averaging over an integral number of grating periods, and were then compared with those of the approximate solutions. Figure 2 displays the ratio of peak intensities as a function of the soliton parameter $\lambda$, and reveals that, for both anomalous and normal dispersion, the approximate solutions work well for small $\lambda$ and large $|K|$, as expected. Even when $|K|$ is not so large $(|K|=4)$, the first-order solutions provide a much better fit than the zeroth-order solutions (dotted line). The zeroth-order solution does not depend on $K$ (and hence $\Delta k$ ), and corresponds to the $\tilde{\gamma}=0$ first-order solution. 

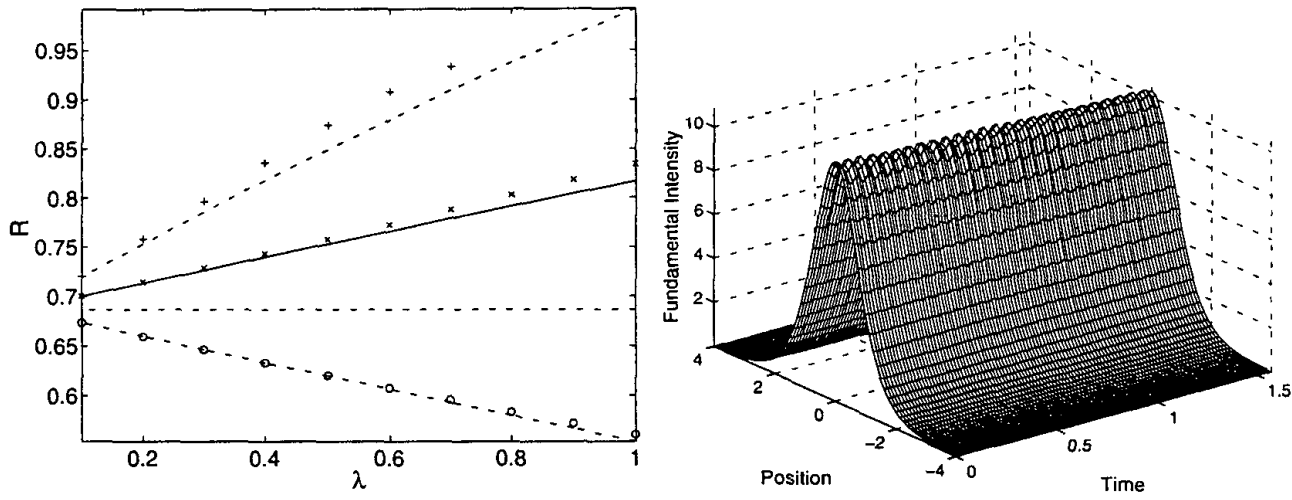

Fig. 2. LEFT: Ratio of peak intensities $R=\tilde{v}^{2}(0) / \tilde{w}^{2}(0)$ versus the soliton parameter $\lambda$, calculated from the first-order solutions with $\tilde{\gamma}=0.0189$ (solid), $\bar{\gamma}=0.0474$ (dot-dashed) and $\tilde{\gamma}=-0.0189$ (dashed), and from the zeroth-order solution $(\dot{\gamma}=0$, dotted). The ratios were also calculated from the average of the evolving field solutions for $K=10$ (crosses), $K=4$ (plusses) and $K=-10$ (circles). In all cases, $\beta=0$. RIGHT: Cubic (sech) bright soliton propagation in the dual grating, showing the evolution of the fundamental intensity. Here $K=100, \beta=0$ and $\lambda=1$.

A given solution of $\tilde{w}$ and $\tilde{v}$ represents a range of physical situations, with different combinations of linear and nonlinear gratings. The propagating solutions shown in Fig. 2 (Left) correspond to a symmetric $\left(d_{0}=0\right)$ nonlinear grating with no linear grating. However other combinations of gratings were simulated and fit just as well to the first-order results.

Now without the linear grating and a DC component of the nonlinear grating, the cubic terms, as a first-order effect, can only be small. However, if the linear grating is present, then the cubic to quadratic ratio can be greatly increased. In fact, the effective quadratic nonlinearity will vanish totally $(\rho=0)$ in certain cases. For realistic physical parameters, both bright and dark solitons still exist as solutions to the average equations [Eq. (2)] when $\rho=0$, due to the cubic terms. If, as in previous work[2,3], the induced cubic nonlinearities are ignored, then no solitons are predicted to exist, since there is no nonlinearity. Once again, a severe test for the validity of these solutions is to launch them into simulations of the field equations. Figure 2 shows the evolution of a bright soliton for $\gamma<0$ that has a sech profile in the fundamental and a vacuum in the second harmonic field. There is no discernible shedding of radiation when the soliton is excited, and the intensities vary only slightly. Similarly, dark tanh solitons were found to propagate stably for $\gamma>0$. We consider modulational stability of dark solitons in another submission.

\section{References}

1. M. Scalora, J. P. Dowling, C. M. Bowden and M. J. Bloemer, "Optical Limiting and Switching of Ultrashort Pulses in Nonlinear Photonic Band Gap Materials," Phys. Rev. Lett. 73, 1368-1371 (1994).

2. M. M. Fejer, G. A. Magel, D. H. Jundt, and R. L. Byer, "Quasi-phase-matched se cond harmonic generation: Tuning and tolerances," IEEE Journal of Quantum Electronics 28, 2631-2654 (1992).

3. T. Suhara and H. Nishihara, "Theoretical analysis of waveguide second-harmonic generation phase matched with uniform and chirped gratings," IEEE Journal of Quantum Electronics 26, 1265-1276 (1990); matching in second harmonic generation in titanium lithium niobate waveguides," in Spie: Integrated Optical Circuit Engineering III 251, 221-228 (1986); D. V. Petrov, "Nonlinear phase shift by cascaded quasi-phase-matched second harmonic generation," Opt. Commun. 131, 102-106 (1996).

4. D. C. Hutchings, "Modulation of the second-order susceptibility in GaAs/AIAs superlattices," Appl. Phys. Lett. 76, 1362-1364 (2000); D. C. Hutchings, private communication.

5. C. B. Clausen, O. Bang and Y. S. Kivshar, "Spatial solitons and induced Kerr effects in quasi-phase-matched quadratic media," Phys. Rev. Lett. 78, 4749-4752 (1997).

6. J. A. Armstrong, N. Bloembergen, J. Ducuing and P. S. Pershan, "Interactions between light waves in a nonlinear dielectric," Phys. Rev. 127, 1918-1939 (1962).

7. O. Bang, "Dynamical equations for wave packets in material with both quadratic and cubic response," J. Opt. Soc. Am. B 14, 51-61 (1997). 